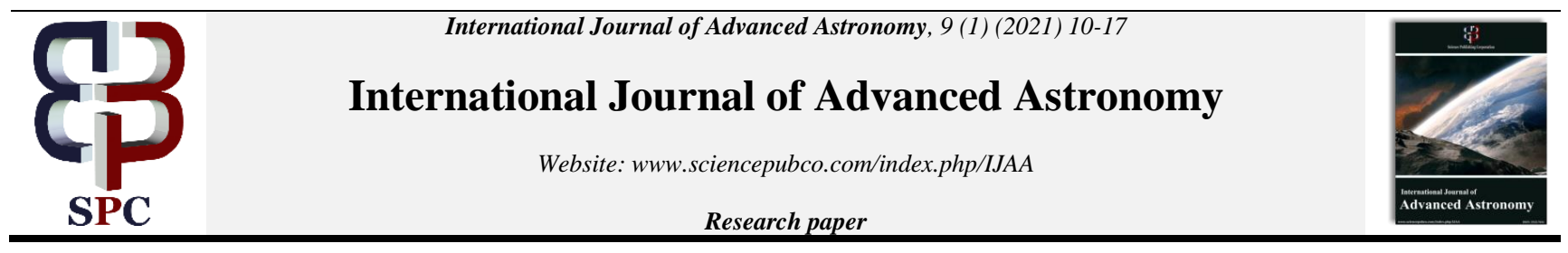

\title{
Diagnostics of interstellar medium parameters on non-discrete timing irregularities of pulsars
}

\author{
E. U. Iyida ${ }^{1,2}$, C.I. Eze ${ }^{1,2}$ \\ ${ }^{1}$ Astronomy and Astrophysics Research Lab., Department of Physics and Astronomy, University of Nigeria, Nsukka \\ ${ }^{2}$ Department of Physics and Astronomy, Faculty of Physical sciences, University of Nigeria, Nsukka, Nigeria \\ *Corresponding author E-mail: evaristus.iyida@unn.edu.ng
}

\begin{abstract}
In this paper, a large homogenous sample of Jodrell Bank Observatory (JBO) radio pulsars was used to investigate the statistical effects of interstellar medium (ISM) parameters: dispersion and rotation measure ( $D M$ and $R M$, respectively) on non-discrete timing irregularities of our sample (whose observed timing activity timescales span over 40 years). This is done by using the correlations between the measured $D M$ and $R M$, and some parameters that have been commonly used to measure non-discrete timing irregularities [timing activity parameter $(A)$, the amount of timing fluctuations absorbed by the cubic term $\left(\sigma_{\mathrm{R} 23}\right)$, measure of pulsar rotational stability $\left(\sigma_{\mathrm{Z}}\right)$ and stability parameter $\left(\Delta_{8}\right)$ ]. Our results show that ISM parameters positively correlate $(r>0.60)$ with the pulsar timing irregularities parameters of our sample. The significant relationships observed are discussed.
\end{abstract}

Keywords: Methods: Statistical; Pulsars: General; Interstellar Medium: General.

\section{Introduction}

Pulsars (highly magnetized, fast rotating neutron stars) are remnants of supernova explosion of $\mathrm{O}$ and $\mathrm{B}$ stars that are created in the collapse of the stellar core. They offer the opportunity to study physics in regimes unattainable in any terrestrial laboratory and provide a powerful probe for exploring the interstellar medium (Lyne \& GrahamSmith, 2005; 2012). Their periods of rotation are remarkably stable such that pulsars are extremely good cosmic clocks (Bell-Burnell, 1977). This clock-like behavior has different applications, for example, testing gravitational theories (see e.g. Kramer et al., 2006; Cameron et al., 2018; Stovall et al., 2018) as well as detection of stochastic background of gravitational waves (Shannon et al., 2015; Arzoumanian et al., 2018). However, the clocks are far from perfect as they slow down with time leading to increase in spin periods $\mathrm{P}$ according to the spin down law (Manchester \& Taylor, 1977) given as

$$
\dot{P}=K P^{2-n}
$$

where the exponent $\mathrm{n}$ is the braking index and $\mathrm{K}$ is a scaling constant that is related to the pulsar moment of inertia and magnetic field structure (Gunn \& Ostriker, 1969). Measurements of the braking index is obtained by differentiating and eliminating the constant K in equation (1) (see e. g. Manchester \& Taylor 1977; Lorimer and Kramer, 2005) to give

$$
n=2-\frac{P \ddot{P}}{\dot{P}^{2}} .
$$

While pulsars possess exceptional rotational stability, large scale timing studies have revealed two distinct types of irregularities in their rotation termed discrete events (macroglitches and microglitches) and non-discrete events (timing noise and white noise) (Hobbs et al., 2010; Yu et al., 2013; Chukwude \& Odo, 2016). Macroglitches are unusual spectacular events usually characterized by recovery of the pulsar spin down rate on wide range of timescales (Flanagan 1990; 1995; Urama 2002; Eya et al., 2017; Eze et al., 2018). Microglitches $\left(|\Delta v / v|<10^{-9}\right.$ ) on the other hand, are not well understood, less attractive and described by variable signatures (Cordes \& Downs, 1985; Chukwude \& Urama, 2010; Eya et al., 2019). Generally, glitches are posited to originate from either the release 
of built up tension within the neutron star's crust by starquakes (Ruderman, 1969), or the unpinning of superfluid vortices from the crustal lattice (Anderson \& Itoh, 1975; Alpar et al., 1984; Melatos et al., 2008).

However, non-discrete type of rotational anomalies called timing noise (TN) is the random, sustained fluctuations in the pulsars' spin parameters implying an auto correlated process on a time-scale of months to years and is generally described by a wide-sense stationary stochastic signal (Groth 1975; Hobbs et al., 2010). It is thought to arise due to changes in the coupling between the neutron star crust and its super-fluid core (Jones 1990) or magnetospheric torque fluctuations (Cheng, 1987, Lyne et al., 2010). On the other hand, White noise (WN) as a manifestation of non-discrete anomaly is characterized by large amplitude scatters in the observed timing residuals (see e.g. D’Alessandro et al., 1993; Chukwude, 2007; Hobbs et al., 2010).

A number of different analytic parameters have been used to measure the amount of non-discrete timing irregularities in pulsars. Ideally, the qualitative measurement of the level of this non-discrete timing irregularities in pulsars can be found from the additional phase residuals left after accounting for its deterministic spin-down (Cordes and Greenstein, 1981; D'Alessandro et al., 1995). Nevertheless, the evolution of timing irregularities parameters with various spin-down parameters have been extensively used for detailed study of pulsar populations (Urama et al., 2006; Chukwude et al., 2010; 2012; Iyida et al., 2018; 2020). Arzoumanian et al. (1994) in order to quantify the amount of non-discrete timing irregularities in pulsars fitted the observed rotational phase of each pulsar of spin frequency $\boldsymbol{v}$ and its first and second time derivatives $\dot{\boldsymbol{v}}$, and $\ddot{\boldsymbol{v}}$ respectively with a function of the Taylor series expansion of the form:

$$
\omega_{(t)}=\omega_{0}+\left(v_{t-t_{0}}\right)+0.5 \dot{v}\left(t-t_{0}\right)^{2}+0.2 \ddot{v}\left(t-t_{0}\right)^{3}+\ldots
$$

where $\boldsymbol{\omega}_{(t)}$ is the phase at a time $\boldsymbol{t}$. They defined the stability parameter $\Delta_{8}$ as

$$
\Delta_{8}=\log \left(\frac{1}{6}|\ddot{v}|\left(10^{8} s\right)^{3}\right) .
$$

Earlier on, Cordes and Helfand (1980) introduced the activity parameter $(A)$ defined as the logarithm of the ratio of the root mean square deviation over a $12 \mathrm{~ms}$ observation for a given pulsar to the same quantity in the crab pulsar over a time span $T$ in days and explicitly given as

$$
A=\log \left[\frac{\sigma_{T N}(m, T)}{\sigma_{T N}(m, T)_{c r a b}}\right]
$$

where $\boldsymbol{m}$ is the order of the polynomial fit to the data, $\sigma_{\mathrm{TN}}(\boldsymbol{m}, \boldsymbol{T})$ is contribution to the gross root mean square phase residuals while $\boldsymbol{\sigma}_{\mathrm{TN}}(\boldsymbol{m}, \boldsymbol{T}) \mathrm{crab}$ is the timing noise of the crab pulsar (in ms). Matsakis et al. (1997) based on the Allan variance of terrestrial clocks introduced pulsar clock stability $\sigma_{Z}(\boldsymbol{\tau})$ which uses the average magnitude of $\ddot{\boldsymbol{v}}$ over short time spans, $T$ and expressed as

$$
\sigma_{Z}(T)=\frac{1}{2 \sqrt{5}}\left[\frac{\sigma_{\ddot{v}}(T)}{v}\right] T^{2}
$$

where $\sigma_{v}{ }^{\prime \prime}$ is the root-mean-square of the measured $\ddot{\boldsymbol{v}}$. Chukwude (2003) proposed the amount of contamination obtained in $\ddot{\boldsymbol{v}}$ from the standard timing activity by all forms of timing fluctuation with

$$
\sigma_{R 23}(T)=\sqrt{\sigma_{R}^{2}(2, T)-\sigma_{R}^{2}(3, T)}
$$

where $\sigma_{R}^{2}(2, T)$ and $\sigma_{R}^{2}(3, T)$ are, respectively, the differences of timing residuals over time $T$ after a second and third-order polynomial fits.

However, a significant analytical parameter for measuring the degree of timing fluctuations in the smooth spindown of pulsars is the root mean square (rms) phase residuals over an observing span of length $T$ after fitting a polynomial of order $m$ whose variance is defined (see e.g. Cordes \& Downs, 1985) as 


$$
\sigma_{R}^{2}(m, T)=\frac{1}{N_{t}} \sum_{i=1}^{N_{t}} R^{2}\left(t_{i}\right)
$$

where $N_{t}$ is the number of observations and $R(t)$ is the residuals of the fit. The difference in the timing residuals is expressed as a quadrature sum of the timing noise and white noise components given in the form (e.g. Cordes \& Downs, 1985; D’Alessandro et al., 1995)

$$
\sigma_{R}^{2}(m, T)=\sigma_{T N}^{2}(m, T)+\sigma_{W N}^{2}
$$

where $\sigma_{T N}^{2}(m, T)$ and $\sigma_{W N}^{2}$ are, respectively, the mean square contributions from timing noise and white noise processes. The mean square white noise fluctuations can be written (e.g. Cordes \& Downs, 1985; D`Alessandro et al., 1995) as

$$
\sigma_{W N}^{2}=\sigma_{M}^{2}+\sigma_{J}^{2}
$$

where $\sigma_{M}^{2}$ and $\sigma_{J}^{2}$ are the mean square contributions from measurement noise and pulse phase jitter, respectively. Previous analyses using the white noise component of Eq. (9) was theoretically presumed to be zero and in this condition, $\sigma_{T N}^{2}(m, T)>>\sigma_{W N}^{2}$ was properly satisfied for small samples (Chukwude, 2007; Chukwude and Urama, 2010). However, a more unbiased method adopted in the current analysis is to assume that the observed pulsar timing activity is composed of both timing and white noise fluctuations. This implies that the calculated pulsar timing properties would be more appropriately considered as the sum of fluctuations produced by the two broad processes. As a result, the amount absorbed by the cubic term is the sum of the timing noise and white noise components (see e.g. Chukwude and Odo, 2016) given as

$$
\sigma_{R 23}(T)=\sigma_{T N}(T)+\sigma_{W N}
$$

where $\sigma_{T N}(T)$ and $\sigma_{W N}$ are, respectively, the rms timing noise and white noise components of $\sigma_{\mathrm{R} 23}(T)$. Similarly, the activity parameter $(A)$ of radio pulsars can be written in the form

$$
A(T)=A_{T N}+A_{W N}
$$

where $A_{\mathrm{TN}}$ and $A_{\mathrm{WN}}$ correspond to the contributions from both TN and WN fluctuations respectively. Following the same argument, the pulsar clock stability parameter $\left(\sigma_{\mathrm{z}}\right)$ can be expressed as

$$
\sigma_{Z}(T)=\sigma_{Z[T N]}(T)+\sigma_{Z[W N]}
$$

where $\sigma_{Z[T N]}(T)$ and $\sigma_{Z[W N]}$ are the components of the parameter from TN and WN, respectively. The dependence of the non-discrete timing irregularities parameters $\left(\sigma_{\mathrm{R} 23}, A\right.$ and $\left.\sigma_{\mathrm{z}}\right)$ on data span length $T$ comes from the non-stationarity of TN fluctuations (e.g. Cordes \& Downs 1985). It follows that $\sigma_{\mathrm{R} 23}(T), A(T)$ and $\sigma_{\mathrm{z}}(T)$ apply only to the case where the contributions from TN strongly dominates over that of $\mathrm{WN}$

However, the electromagnetic radiations that are produced by a rotating pulsar interact with the free electrons in the interstellar medium (ISM), thus, affecting the waves to travel at frequency dependent velocities. As the pulsar signal travels through the ISM, the overall column density of free electrons has dispersive effects on the broadband pulses. The group velocity of the pulse train is

$$
v_{g}=c \sqrt{1-\frac{v_{P}^{2}}{v^{2}}}
$$

where $c$ is the speed of light, $v$ is the observing frequency and $v_{p}$ is the plasma frequency given by

$$
v_{P}=\sqrt{\frac{n_{e} e^{2}}{\pi m_{e}}} \approx 10^{4} \mathrm{~Hz} \sqrt{\frac{n_{e}}{1 \mathrm{~cm}^{-3}}}
$$

$e$ is the electron charge, $m_{e}$ is the electron mass and $n_{e}$ is the electron density in the relevant direction in the sky. 
The Dispersion Measure $(D M)$ which is a measure of the integrated column density of free electrons along some line of sight is written as

$$
D M=\int_{0}^{d} n_{e} d l
$$

where $d$ is the distance to the pulsar. These radiations are dispersed by the ionized content of ISM as they travel. Consequently, this leads to a delay in the arrival time of the pulses due to the dispersion measure at two different observing frequencies $\left(v^{-a}\right.$ and $\left.v^{-b}\right)$ (Lorimer \& Kramer 2005; Lyne \& Graham-Smith, 2012) given as

$$
t_{D M}(v)=\frac{e^{2}}{2 \pi m_{e} v^{2}} D M \approx 4.15 \times 10^{3}\left(v_{a}^{-2}-v_{a}^{-2}\right) \times D M
$$

The observational consequence of the dispersive delay is that a pulsar observed with a broad frequency band will show pulses that arrive later at lower frequency than at higher frequency following the $v^{-2}$ law. By observing pulsars at separate frequency bands, the dispersion measure can be measured at each observing epoch. However, as the radiations travel through a highly magnetized medium, Faraday rotation of the plane of polarization occurs and measurement of the position angle over a wide range of frequencies gives the corresponding rotation measure $(R M)$ given (e.g. Lorimer \& Kramer 2005) as

$$
R M=\frac{e^{3}}{2 \pi m_{e}^{2} c^{4}} \int_{0}^{d} n_{e} B_{\|} d l
$$

$B_{\|}$is the magnetic field strength along the line of sight weighted by electron density. Evidently, the dispersion measure $(D M)$ and rotation measure $(R M)$ are dependent on the electron number density of the ISM and are extrinsic to pulsar rotational irregularities. It follows, therefore that both $D M$ and $R M$, which characterize the intervening ISM are expected to be dependent on spin frequency and its derivatives, which describe rotational irregularities. Timing irregularities introduce variations in spin frequency and its derivatives which becomes problematic in the long-term measurement of pulse's arrival time. In this paper, we explore the contributions of interstellar medium parameters $(D M$ and $R M)$ on timing irregularities and the intrinsic evolution of radio pulsars.

\section{Data analysis and results}

The data used in the analysis is from Jodrell Bank Observatory (JBO) radio pulsars with significant measurements of non-discrete timing irregularities parameters estimated from radio timing data with time span length of over four decades (see e.g. Hobbs et al.2004; 2010 for more details). This dataset is dominated by either TN or WN. The activity parameter was computed using equation (12) on the assumption that the method used effectively whitened the timing residuals of the pulsars in the sample while 366 objects with complete information on pulsar clock stability parameter $\sigma_{\mathrm{Z}}(\tau)$ were compiled from literature Hobbs et al. (2010). Also, the timing noise statistic was calculated from the information published in Hobbs et al. (2010) using equation (11). The corresponding rotation measure (RM) and dispersion measure (DM) were all extracted from the Australian Telescope National Facility (ATNF) Pulsar catalogue. We used both analytic and statistical methods to analyze the data collected for this study. The statistical analyses indicate the strength of relationship between pairs of variables with the aim to understand which among the independent variables are related to the dependent variable and to explore the forms of their relationships. This method enabled us to calculate the correlation coefficient $r$, which provides the strength of correlation between the parameters.

In order to investigate the distribution pattern of dispersion and rotation measure, Figure 1 shows the distribution of these parameters on logarithmic scales. It is evident from Fig. 1a that, the spread in the values of dispersion measure is about 5 orders of magnitude $\left(\sim 4-1000 \mathrm{~cm}^{-3} \mathrm{pc}\right)$ with a mean value of $\sim 174 \mathrm{~cm}^{-3} \mathrm{pc}$. The distribution is unimodal and follows a near normal distribution with about $50 \%$ of the objects having dispersion measure below the mean value.

Similarly, the distribution of the magnitude of the rotation measure of our sample is shown in Figure 1b. the rotation measure ranges from $\sim-320$ to $2250 \mathrm{radm}^{-2}$. This is a wide spread ( $\sim 4$ orders of magnitude with a mean value of $\sim 72 \mathrm{radm}^{-2}$. The distribution of rotation (irrespective of sign) is skewed negatively with about 155 pulsars having rotation measure values above the mean value. 

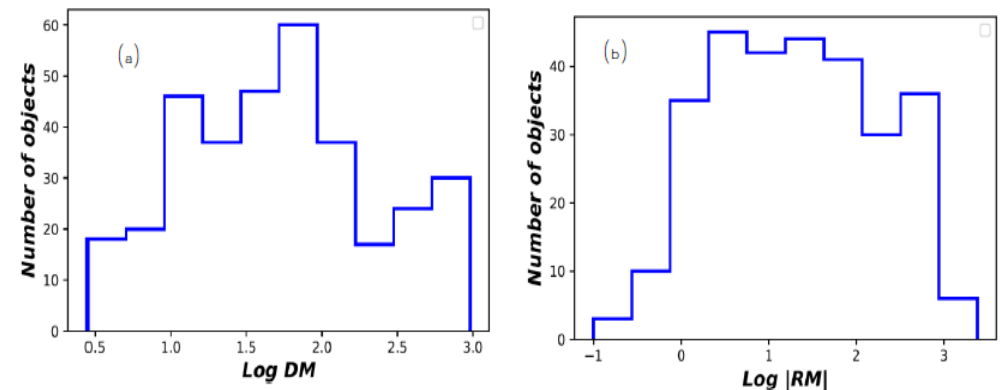

Fig. 1: Histogram Showing the Distributions of the Dispersion and Rotation Measure of Our Pulsar Sample.

Figure 2(a-d) shows the scatter plots of $\log |\mathrm{RM}|$ against the timing irregularities parameters for the 523 radio pulsars. The scatter in the rotation measure data for any timing irregularities parameters is quite large and with up to 5 orders of magnitude. However, it is striking to observe that the rotation measure (irrespective of sign) appear, on average, to increase with timing irregularities parameters. The figures actually show an upward sloping trend with closely clustered scatter points depicting that pulsars with large values of rotation measure have large values of timing irregularities parameters. Regression analyses of our data yield positive correlation $(r \sim 0.68)$ for the variables being studied. The exact forms of their relationship are:

$\log |R M|=(0.97 \pm 0.20) A+(-1.05 \pm 0.01)$

$\log |R M|=(1.02 \pm 0.20) \log \sigma_{23}+(-1.00 \pm 0.01)$

$\log |R M|=(1.31 \pm 0.20) \Delta_{8}+(-1.03 \pm 0.01)$

and

$\log |R M|=(0.61 \pm 0.20) \log \sigma_{Z}+(-1.01 \pm 0.01)$
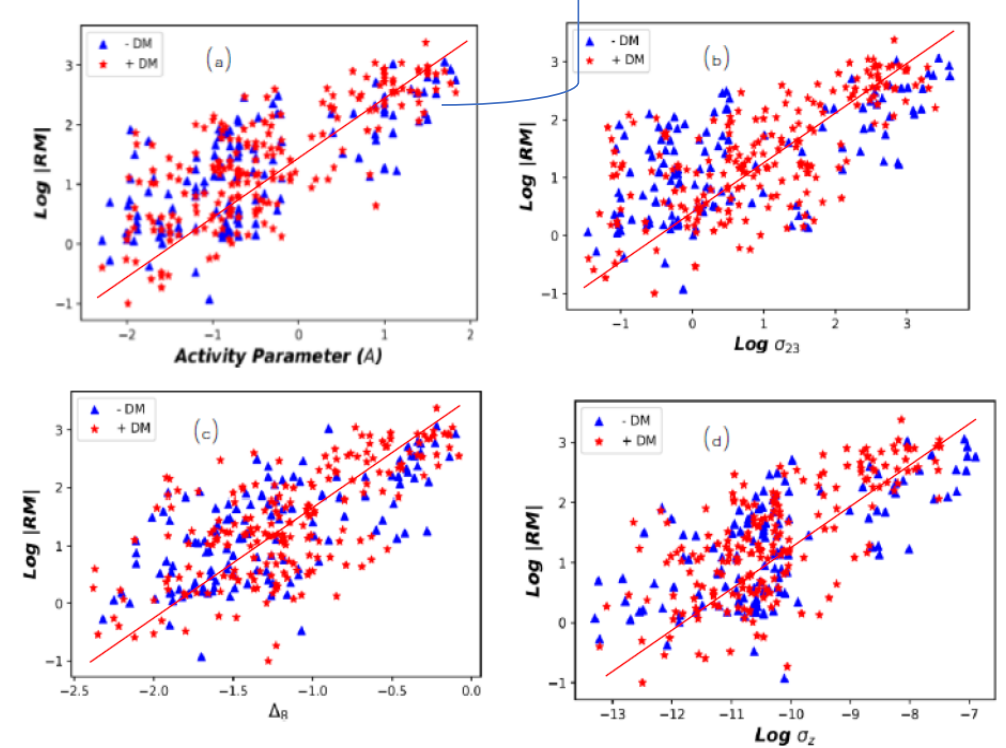

Fig. 2: Scatter Plots Showing the Relationships between Absolute Magnitude of Rotation Measure Against NonDiscrete Timing Irregularities Parameter.

In addition, we find a dependence of timing irregularities parameters on dispersion measure for our sample. Figure $3(\mathrm{a}-\mathrm{d})$ shows the scatter plot of dispersion measure as a function of timing irregularities parameters. The plots are characterized by large amplitude ( $\sim$ up to 5 orders of magnitude) scatter in both dispersion measure and timing irregularities parameters data. However, superimposed on this scatter is a striking trend which suggests possible relationship between the parameters, in which pulsars with small timing irregularities parameters on average, have small dispersion measure. It is found that the dispersion measure is positively correlated (correlation coefficient $r$ $\sim 0.72$ ) with timing irregularities parameters. The implication of this is that pulsars with large dispersion measure exhibit more timing irregularities. The large amplitude scatter observed in current sample is not entirely unexpected given the large-scale inhomogeneity in electron density distribution in the interstellar medium and uncertainty in pulsar rotation periods (see e.g. Cordes et al., 1985; Cordes and Chernoff, 1997). Regression analyses of our data give positive correlations $(r>0.68)$ between dispersion measure and timing irregularities parameters. The best fits to our data yield regression equations expressed as follows: 

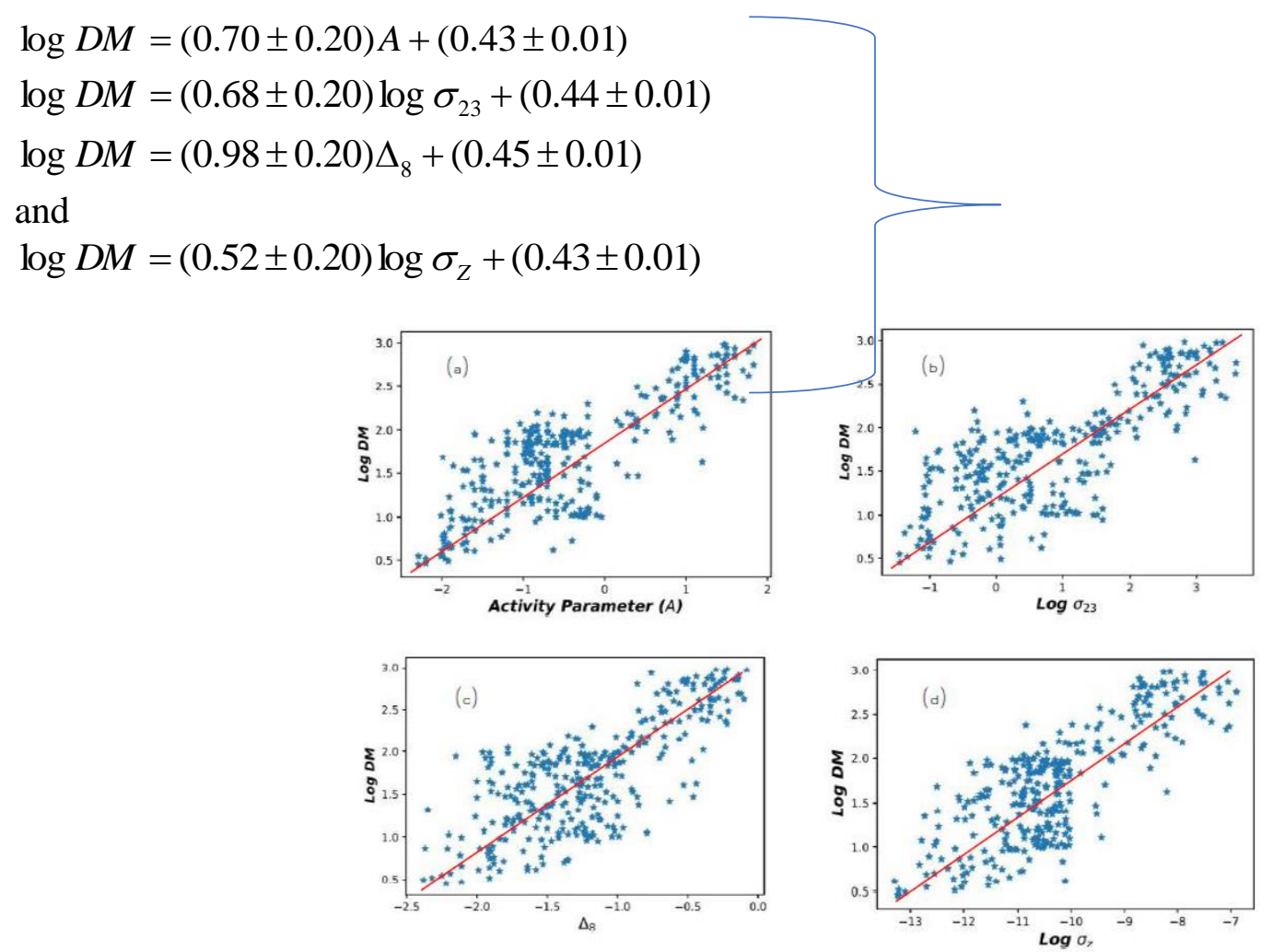

Fig. 3: Scatter Plots Showing the Relationships Between Dispersion Measure Against Non-Discrete Timing Irregularities Parameters

\section{Discussion}

The result of the statistical analysis of a large data base of rotation powered pulsars with significant measurements of interstellar medium parameters and their effects on the timing irregularities parameters has been presented. The ISM consists of neutral and molecular hydrogen gas, ionized gas (HII), dust grains and magnetic field. It is a diffuse medium of gas and dust between stars. It is widely believed that the distribution of electron density in the ISM is very inhomogeneous (Backer et al. 1993; Hobbs et al. 2004; Lorimer \& Kramer, 2005; Hobbs et al., 2010). This inhomogeneity in the electron content of the ISM might be responsible for the observed large scatter in DM and RM data. If a pulsar is associated with HII region or supernova remnant, it will invariably be associated with large dispersion measure/rotation measure. This could account for the large scatter observed in both DM and RM. The trend observed in the relationship between the interstellar medium (DM and RM) parameters and the radio pulsar timing irregularities parameters is remarkable. This is because the interstellar medium is expected to significantly affect the intrinsic spin-down of radio pulsars and thus, the timing irregularities. The significance of the relationship is that it strongly supports the widely accepted theory for pulsar rotation and evolution.

\section{Conclusion}

We have revealed, using a large homogenous sample of radio pulsars with accurately measured interstellar medium parameters that the timing irregularities of pulsars on a wide range of timescales is sufficiently dominated by fluctuations. Specifically, our results show that interstellar medium parameters of radio pulsars in our sample, obtained from standard measurements, are significantly correlated with timing irregularities parameters. The observed large scatter in $D M$ and $R M$ against the timing irregularities parameters data highlights the complex nature of the electron content distribution in the ISM and the large dispersion in the magnitude timing irregularities of pulsars in our sample.

\section{Acknowledgement}

We are thankful to the referee for very useful comments and suggestions which helped to improve this paper. 


\section{References}

[1] Anderson, P.W., Itoh, N. (1975). Pulsar glitches and restlessness as a hard superfluidity phenomenon. Nature, 256,2527 https://doi.org/10.1038/256025a0.

[2] Alpar, M.A., Anderson, P.W., Pines, D., Shaham, J. (1984). Vortex Creep and the Internal Temperature of Neutron Stars. I - General theory. Astrophysical Journal. 276, 325- 334. https://doi.org/10.1086/161616.

[3] Arzoumanian, Z., Baker, P.T., Brazier, A., Burke-Spolaor, S., Chamberlin, S.J., Chatterjee, S., et al. (2018) The NANOGrav 11 Year Dataset: Pulsar Timing Constraints on the Stochastic Gravitational Wave Background. Astrophysical Journal. 859, 47. https://doi.org/10.3847/1538-4357/aabd3b.

[4] Arzoumanian, Z., Nice, D.J., Taylor, J.H. and Thorsett, S.E. (1994) Timing Behavior of 96 Radio Pulsars. Astrophysical Journal. 422, 671-680. https://doi.org/10.1086/173760.

[5] Backer, D.C., Foster, R.S. and Sallmen, S. (1993). A Second companion of the millisecond pulsar 1620-26. Nature. 365, 817 -819. https://doi.org/10.1038/365817a0.

[6] Bell-Burnell, S. J. (1977). Petit Four. Annals of the New York Academy of Sciences, 302, 685 https://doi.org/10.1111/j.1749-6632.1977.tb37085.x.

[7] Cameron, A.D., Champion, D.J., Kramer, M., Bailes, M., Barr, E.D., Bassa, C.G., et al. (2018) The High Timing Resolution Universe Pulsar Survey-XIII. PSR J1757-1854, the Most Accelerated Binary Pulsar. Mont. Not. of the Royal Astron. Soci. Letters, 475, L57-L61. https://doi.org/10.1093/mnrasl/sly003.

[8] Cheng, K.S. (1987). Outer Magnetospheric Fluctuations and Pulsar Timing Noise. Astrophysical Journal. 321, $799-804$. https://doi.org/10.1086/165672.

[9] Chukwude, A.E. (2003). On the Statistical Implication of Timing Noise for Pulsar Braking Index. Astronomy \& Astrophysics. 406, 667-671. https://doi.org/10.1051/0004-6361:20030789.

[10] Chukwude, A.E., Baiden, A.A. and Onuchukwu, C.C. (2010). Measurements of Radio Pulsar Breaking Indices. Astronomy \& Astrophysics, 515, Article No. A21. https://doi.org/10.1051/0004-6361/200911634.

[11] Chukwude, A.E. (2007). A Statistical Analysis of Radio Pulsar Timing Noise. Chinese Journal of Astronomy and As trophysics. 7, 521-530. https://doi.org/10.1088/1009-9271/7/4/08.

[12] Chukwude, A.E. and Odo, F.C. (2016). Assessing the Effects of Timing Irregularities on Radio Pulsars Anomalous Braking Indices. Research in Astronomy and Astrophysics. 16, 150. https://doi.org/10.1088/1674-4527/16/10/150.

[13] Chukwude, A.E. and Butcner, S. (2012). Dual Spin-Down States of the Pulsar J1001-5507. Astrophysical Journal. 745, 40-47. https://doi.org/10.1088/0004-637X/745/1/40.

[14] Chukwude, A. E., Urama, S. O. (2010). Observations of Microglitches in Hartebeesthoek Radio Astronomy Observatory Radio Pulsars. Mont. Not. of the Royal Astron. Soci., 406, 1907-1917. https://doi.org/10.1111/j.13652966.2010.16789.x.

[15] Cordes, J.M. and Greenstein, G.S. (1981). Pulsar Timing IV: Physical Models for Timing Noise Models. Astrophysical Journal. 245, 1060-1079. https://doi.org/10.1086/158883.

[16] Cordes, J.M. and Chernoff, D.F. (1997) Neutron Star Population Dynamics. I. Millisecond Pulsars. Astrophysical Journal. 482, 971 https://doi.org/10.1086/304179.

[17] Cordes, J.M. and Helfand, D.J. (1980) Pulsar Timing. III. Timing Noise of 50 Pulsars. Astrophysical Journal. 239, 640650. https://doi.org/10.1086/158150.

[18] Cordes, J.M., Weisberg, J.M. and Boriakoff, V. (1985). Small-Scale Electron Density Turbulence in the Interstellar Medium. Astrophysical Journal. 288, 221-247. https://doi.org/10.1086/162784.

[19] Cordes, J. M. and Downs, G. S. (1985). JPL Pulsar Timing observations. III. Pulsar rotation fluctuations. Astrophysical Journal Suppl. 59, 343-382 https://doi.org/10.1086/191076.

[20] D’Alessandro, F., Mc Culloh, P.M., Hamilton, P.A. and Deshpande, A.A. (1995). The Timing Noise of 45 Southern Pulsars. Mont. Notic. of the Royal Astro. Soc., 277, 1033-1046. https://doi.org/10.1093/mnras/277.3.1033.

[21] D’Alessandro, F., Mc Culloch, P.M., King, E.A., Hamilton, P.A. and McConnell, D. (1993) Timing Observations of Southern Pulsars: - 1987 to 1991. Mont. Not. of the Royal Astron. Soci., $261,883$. https://doi.org/10.1093/mnras/261.4.883.

[22] Groth, E. J. (1975). Timing of the Crab Pulsar II. Method of Analysis. Astrophysical Journal. $29,443-451$. https://doi.org/10.1086/190353.

[23] Gunn, J. E. and Ostriker, J. P. (1969). Magnetic Dipole Radiation from Pulsars. Nature, 221, $454-456$ https://doi.org/10.1038/221454a0.

[24] Kramer, M., Stairs, I.H., Manchester, R.N., McLaughlin, M.A., Lyne, A.G., Ferdman, R.D., et al. (2006) Tests of General Relativity from Timing the Double Pulsar. Science, 314, 97-102. https://doi.org/10.1126/science.1132305.

[25] Eya, I.O., Urama, J.O. and Chukwude, A.E. (2019). On the Distributions of Pulsar Glitch Sizes and Inter-Glitch Time Intervals. Research in Astronomy and Astrophysics. 19, 89. https://doi.org/10.1088/1674-4527/19/6/89.

[26] Eya, I.O., Urama, J.O. and Chukwude, A.E. (2017). Angulsar Momentum Transfer and Fractional Moment of Inertia in Pulsar Glitches. Astrophysical Journal. 840, 56. https://doi.org/10.3847/1538-4357/aa6b55.

[27] Eze, C.I., Iyida, E.U. Odo, F.C. and Urama, J.O. (2018). Statistical Study of Glitch Behaviours of Glitching Pulsars. International Journal of Astrophysics and Space Science. 6(4), 62-72. https://doi.org/10.11648/j.ijass.20180604.11.

[28] Flanagan, C.S. (1990). A Brief History of Pulsar Time. Nature. 345, 416-417 https://doi.org/10.1038/345416a0 https://doi.org/10.1038/345416a0.

[29] Flanagan, C.S. (1995) Unpublished Ph.D Thesis, Rhodes University of Grahamstown, South Africa. 
[30] Hobbs, G., Lyne, A.G. and Kramar, M. (2010) An Analysis of the Timing Irregularities for 366 Pulsars. Mont. Not. of the Royal Astro. Soc., 402, 1027-1048. https://doi.org/10.1111/j.1365-2966.2009.15938.x.

[31] Hobbs, G., Lyne, A. G., and Kramar M. (2010). An analysis of the timing irregularities for 366 pulsars. Mon. Not. R. Astron. Soc., 402, 1027-1048 https://doi.org/10.1111/j.1365-2966.2009.15938.x.

[32] Hobbs, G., Lyne, A. G., Kramar, M., Martin, C.E. and Jordan, C. (2004). Long-term observations of 374 Pulsars. Mon. Not. R. Astron. Soc., 353, 1311-1344 https://doi.org/10.1111/j.1365-2966.2004.08157.x.

[33] Iyida, E.U., Eze, C.I., Eya, I.O., Chukwude, A.E. (2018) On the Effects of Spin of Properties on Timing Noise Parameters of Rotation-Powered Pulsars. International Journal of Astrophysics and Space Science. 6, 101-107.

[34] Iyida, E.U., Eze, C.I. and Eya, I.O. (2020) On Strong Correlations between Timing Irregularities and Surface Magnetic Field of Rotation-Powered Pulsars. International Journal of Astronomy and Astrophysics. 10, 346-355. https://doi.org/10.4236/ijaa.2020.104019.

[35] Jones, P. B. (1990). The Generation of Timing Noise by Superfluid Rotation in Pulsars. Mont. Not. of the Royal Astro. Soc., 246, 364-369

[36] Kramer, M., Lyne, A.G., O’Brien, J.T., Jordan, C.A. and Lorimer, D.R. (2006). Periodically Active Pulsar Giving Insight into Magnetospheric Physics. Science, 312, 549-551. https://doi.org/10.1126/science.1124060.

[37] Lyne, A.G., Hobbs, G., Kramer, M. and Stairs, B. (2010). Switched Magnetospheric Regulation of Pulsar Spin-Down. Science, 329, 408-412. https://doi.org/10.1126/science.1186683.

[38] Lyne, A. G., Graham-Smith, F. (2012). Pulsar Astronomy, Cambridge University Press University Press

[39] Lyne, A. G. and Graham-Smith, F. (2005). Pulsar Astronomy, Cambridge, UK: Cambridge University Press.

[40] Lyne, A. G. and Graham-Smith, F. (2005). Pulsar Astronomy, Cambridge, UK: Cambridge University Press.

[41] Lorimer, D.R. and Kramar, M. (2005) Handbook of Pulsar Astronomy. Cambridge University Press, Cambridge.

[42] Manchester, R.N. and Taylor, J.H. (1977). Pulsars. W.H. Freeman and Company, San Francisco.

[43] Matsakis, D.N., Taylor, J.H. and Eubanks, T.M. (1997). A Statistic for Describing Pulsar and Clock Stabilities. Astronomy \& Astrophysics. 326, 924-928.

[44] Melatos, A., Peralta, C. and Wyithe, J.S.B. (2008). Avalanche Dynamics of Radio Pulsar Glitches. Astrophysical Journal. 672, 1103-1118. https://doi.org/10.1086/523349.

[45] Ruderman, M. (1969). Neutron Starquakes and Pulsar Periods. Nature, 223, 579-598 https://doi.org/10.1038/223597b0

[46] Shannon, R.M., Ravi, V., Lentati, L.T., Lasky, P.D., Hobbs, G., Kerr, M., et al. (2015). Gravitational Waves from Binary Supermassive Blackhole Missing in Pulsar Observations. Science, 349, 1522-1525. https://doi.org/10.1126/science.aab1910.

[47] Stovall K., Freire, P, Chatterjee, P., et al., (2018). PALFA discovery of a highly relativistic double neutron star binary. Astrophysical Journal Letters. 854 (2), L22 https://doi.org/10.3847/2041-8213/aaad06.

[48] Urama, J.O. (2002) Glitch Monitoring in PSR B1046-58 and B1737-30. Mont. Not. of the Royal Astro. Soc., 330, 5862. https://doi.org/10.1046/j.1365-8711.2002.05099.x.

[49] Urama, J.O., Link, B. and Weisberg, J.M. (2006) A Strong-Correlation in Radio Pulsars with Implications for Torque Variations. Mont. Not. of the Royal Astron. Soc. Letters, 370, L76-L79. https://doi.org/10.1111/j.17453933.2006.00192.x.

[50] Yu, M., Manchester, R.N., Hobbs, G., Johnston, S., Kaspi, V.M., Keith, M., et al. (2013) Detection of 107 Glitches in 36 Southern Pulsars. Mont. Not. of the Royal Astro. Soc., 429, 688-724. https://doi.org/10.1093/mnras/sts366. 